\title{
ENSEÑANZA Y APRENDIZAJE DEL INGLÉS Y LAS POBREZAS IMPERCEPTIBLES \\ EFL TEACHING AND SUBTLE POVERTIES
}

\author{
Volumen 11, Número 3 \\ Setiembre-Diciembre
}

pp. 1-30

Este número se publicó el 15 de diciembre de 2011

Gustavo Córdoba González

La revista está indexada en los directorios:

LATINDEX, REDALYC, IRESIE, CLASE, DIALNET, DOAJ, E-REVIST@S,

La revista está incluida en los sitios:

REDIE, RINACE, OEI, MAESTROTECA, PREAL, HUASCARAN, CLASCO 


\title{
ENSEÑANZA Y APRENDIZAJE DEL INGLÉS Y LAS POBREZAS IMPERCEPTIBLES \\ EFL TEACHING AND SUBTLE POVERTIES
}

\section{Gustavo Córdoba González}

\begin{abstract}
Resumen: La política económica-educativa desplegada en las últimas dos décadas en Costa Rica ha dispuesto que la educación, principalmente pública, se concentre en preparar la mano de obra que los sectores industria y comercio, especialmente los relacionados con la Inversión Extranjera Directa, requieren. Dentro de este marco de acción se resimbolizó el valor social del aprendizaje del inglés en este país y se le asignaron poderes que antes esta lengua no tenía, como el de facilitar la consecución de una plaza laboral mejor pagada que en los sectores productivos tradicionales, ruta dentro de la que se abandona la pobreza material. El propósito de este ensayo es analizar, desde la perspectiva del Bachillerato y Licenciatura en la enseñanza del inglés de la Sede de Occidente, la idea de pobreza dentro de la que está inmersa la política educativa y sobre la cual se resimbolizó la enseñanza y el aprendizaje del inglés. Este análisis se aproxima desde los Derechos Humanos Fundamentales y desde los postulados dentro de los que la universidad pública se propone contribuir con las transformaciones que el país necesita para el logro del bien común. Se concluye que si una actividad académica, como la enseñanza del inglés, se encierra en una idea reducida de pobreza en el afán de contribuir a la mejoría de la situación socioeconómica de individuos, peligra reproducir otros tipos de pobrezas que no son tan perceptibles pero que, desde los Derechos Humanos Fundamentales y los postulados de la universidad pública, van en detrimento de toda la sociedad.
\end{abstract}

Palabras clave: POLITICA EDUCATIVA, POLÍTICA ECONÓMICA, POBREZA, DERECHOS HUMANOS FUNDAMENTALES, ENSEÑANZA APRENDIZAJE DEL INGLÉS

\begin{abstract}
In the last two decades, Costa Rican educational and economic policies have proposed public education as the means to prepare the labor force that industry, commerce, and especially foreign investment sectors require. English language teaching and learning resymbolized its social value as a concomitant consequence of those policies; also, people assigned new powers to this language, as the power of getting better paid jobs than those traditionally offered to middle and lower social classes. Consequently, job finding is believed to be the way in which a person can cross poverty borders. The objective of this article is to analyze the idea of poverty in which educational policies make sense for the general public, and in which English language teaching is perceived to have those powers and new values. This analysis is thought from the "Bachillerato y Licenciatura en la enseñanza del ingles" perspective. Also, the analysis is approached from Fundamental Human Rights and from the public university goals that command to contribute with the countries needed transformations in order to reach common wealth. The conclusion goes around the idea that if an academic activity, as English language teaching, is enclosed within a narrow poverty idea, in order to contribute with individuals' economic improvement; then, the academic activity is in the risk of reproducing other poverties which may not be very perceptible in daily life activities but that they are harmful and counterproductive for the common wealth.
\end{abstract}

Keywords: EDUCATION POLICIES - EFL (English as a Foreign Language) - EIL (English as an International Language) - ESL (English as an International Language) - FUNADMENTAL HUMAN RIGHTS - POVERTY

\footnotetext{
${ }^{1}$ Magíster en Educación con mención en el aprendizaje del inglés de la Universidad Nacional de Costa Rica. Docente e investigador de la Universidad de Costa Rica, Sede de Occidente, labora en el Bachillerato y Licenciatura en la enseñanza del inglés de la Sede de Occidente.
}

Dirección electrónica: tavocor@gmail.com

Artículo recibido: 10 de mayo, 2011

Aprobado: 10 de noviembre, 2011 
La universidad del Estado es la más eminente institución nacional.

Es la llamada a orientar la educación pública en todos sus grados; la llamada a satisfacer todas las exigencias de la cultura superior... el crisol de los estadistas y los conductores de la opinión pública consciente. Es siempre la promesa de tiempos mejores. Esta es la universidad como institución, la del Estado; le atribuimos todos estos valores determinantemente, porque es a ésta a la que todos tenemos derecho de exigírselos...

Galdames

\section{Introducción}

Desde el siglo XIX con la fundación de la Casa de Santo Tomás y la apertura de economía al mercado transnacional, la enseñanza aprendizaje del inglés como actividad político-económica y académica ha acompañado la creación del Estado costarricense. A través de la lengua inglesa se fue fortaleciendo el Estado liberal pues las actividades de exportación y construcción de infraestructura a cargo de compañías extranjeras requerían el conocimiento del inglés para comunicarse con la clase trabajadora. Un claro ejemplo de esta relación entre lengua inglesa y actividad político-económica fue la construcción del ferrocarril al Atlántico costarricense a cargo de la United Fruit Company. De acuerdo con Chomsky

in the late nineteenth century U.S.-based companies began to build railroads and plant bananas in Limón, recruiting workers primarily from Jamaica. Thus the society which developed in Limón was an English-speaking enclave of white North American managers and black Jamaican workers, with a culture and history quite distinct from the rest of Costa Rica, although inevitably intertwined. (1994, p. 837)

Así, entre otras justificaciones, el traer trabajadores de origen jamaicano para desarrollar el ferrocarril y más tarde las plantaciones de banano fue en gran medida una razón pensada en las facilidades comunicativas obvias que norteamericanos y jamaicanos compartían a través del inglés.

Durante todo ese período de construcción previa de la segunda República de Costa Rica, los limitados procesos educativos en la enseñanza y aprendizaje del inglés en primaria y secundaria se concentraron en el valle central (Cfr. Salazar, 2003, y Quesada 1997) y en la zona Atlántica (principalmente en la provincia de Limón). Sobre los procesos educativos en el Caribe costarricense, muy poco ha sido reconocido por la historia nacional pues la blanquitud del supuesto ser costarricense ha predominado y privilegiado el estudio valle-centrista blanco. Sin embargo, la historia registra que las primeras escuelas en donde todas las clases 
se enseñaban en inglés estaban ubicadas en Limón. En este sentido, Castillo explica en su trabajo doctoral que

With the goal of returning home in mind, the Afro-Caribbeans established schools to educate their children in English. These schools focused on fundamental of arithmetic and the English language [...]. Because classes were conducted in English, these schools were known as the English schools. (Gayle: Mattis, White, personal communication, June, 1997). By 1927, thirty-three of these private schools existed in Limón and enrolled 1,500 students, most of them English-speaking Afro-Caribbean of Jamaican descent. (1998, p.4)

La presencia de estas escuelas en el país ya empezaba a vislumbrar la importancia que el inglés tendría para estás comunidades afrocaribeñas; pero también, para la política económica del país. Lamentablemente, por razones de espacio y complejidad, el análisis de la educación en lengua inglesa dado en Limón no puede ser abordado en este artículo.

En cuanto al proceso de enseñanza y aprendizaje del inglés desde la noción valle centrista blanca, esta lengua se configuró inicialmente como un privilegio al que sólo las clases sociales más solventes tenían acceso. La creación de Casa de Santo Tomas marcó no sólo el inicio de la educación formal en el aprendizaje del inglés; también marcó un precedente histórico en cuanto a la distinción sobre el uso y la importancia que el inglés ha tenido a nivel nacional entre todos los actores sociales. Como lo indica Marín (2004, p. 48),

En este momento histórico, el inglés se propuso como una rama electiva para los estudiantes de ese centro de estudio. La enseñanza de las lenguas, entre las que se señalan el latín, el francés y el inglés, se impartía en una hora y media por la mañana y el mismo tiempo en la tarde. En aquella época interesaba que los estudiantes conocieran la gramática y las conjugaciones verbales.

Cabe recalcar que la población estudiantil de aquel centro estaba conformada por los hijos de familias adineradas. Era muy común que los hijos de estas familias realizaran estudios en las universidades de Europa y los Estados Unidos.

En consecuencia, a finales del siglo XIX, la importancia del inglés radicó principalmente en la posibilidad de tener acceso a materiales educativos que permitiera una formación académica de élite. Para la clase dominante costarricense, el inglés era un medio 
instrumental que les permitía mantenerse al tanto y disponer del conocimiento producido en las potencias mundiales. Dicho de otro modo, el inglés se configuró como un medio de acceso a información muy importante.

Con el pasar de los años, las pugnas sociales costarricenses por la igualdad y el derecho a una educación pública y gratuita hacen que la enseñanza del inglés se empiece a democratizar pero bajo perspectivas sociales, económicas y político-educativas distintas a la que la clase dominante había dado razón de ser. Muy por el contrario a la filosofía de enseñanza y el aprendizaje del inglés para el acceso a información y conocimiento de élite mundial, la "democratización" del inglés nace bajo una perspectiva de dinamización del mercado nacional en el escenario de una economía transnacional. A través de la enseñanza y el aprendizaje masivo del inglés se buscó (y ha buscado) la satisfacción de demandas laborales dictadas por el capital transnacional. Como resultado, el acceso a diversas fuentes de empleo (técnico-profesional) se convierte en el slogan discursivo de la agenda políticoeducativa del país. En este contexto político-económico, la enseñanza y el aprendizaje del inglés ya no son actividades educativas exclusivas de las clases ricas. Sin embargo, las razones ideológicas y discursivas sobre su importancia y necesidad se sentaron en concepciones educativas, laborales y sociales muy distintas a las de la clase dominante le dio, manteniendo el orden clasista dentro de los usos y necesidades que el inglés posibilita.

A mitad del siglo XX las repercusiones educativas a nivel universitario en el campo de la enseñanza y el aprendizaje del inglés no se hicieron esperar. De acuerdo con Córdoba, Coto y Ramírez

Hasta ese momento, las personas que impartían las clases de inglés en secundaria eran extranjeros (los cuales enseñaban su lengua materna) o nacionales (quienes aprendieron el idioma mientras estudiaban en el exterior) que carecían de la preparación pedagógica necesaria para ejercer en el campo educativo. Probablemente, y debido a esta situación, la Universidad de Costa Rica ofreció por primera vez en 1954 cursos de capacitación para los profesores de inglés. (2005, p. 4)

Pero este proceso de capacitación sólo podía incorporar cuestiones elementales de enseñanza y aprendizaje ya que los cursos impartidos pretendían de forma aislada dar una formación generalísima en el campo de la pedagogía. Por tal razón, en "1957 se creó la carrera de Enseñanza del inglés en la Facultad de Educación de la Universidad de Costa 
Rica" (Córdoba, Coto y Ramírez, 2005, p. 4). En este sentido, los procesos de democratización de la enseñanza y aprendizaje del inglés generaron que la profesionalización de la planilla docente experimentara un importante cambio en la demanda de profesores de inglés. La universidad, entonces, se encontró llamada a atender las necesidades socioeducativas que se empezaron a dar dentro y fuera del valle central costarricense.

Pero no es sino hasta bien entrada la segunda mitad del siglo XX, que la dinámica económica mundial hace que un país como Costa Rica masifique la enseñanza del inglés a nivel nacional. Se inicia un proceso de apertura e incorporación de la enseñanza y aprendizaje del inglés en los planes de estudio de diversos colegios ${ }^{2}$. Al apostarle Costa Rica a un modelo económico basado en la inversión extranjera directa (IED), las corporaciones transnacionales se empiezan a instalar buscando condiciones de operación que les permitieran mejorar sus capitales en donde el inglés viene a jugar un papel clave.

En 1997 se declara la materia inglés obligatoria para Primer y Segundo Ciclos de la Educación General Básica y en 1998 se inicia la enseñanza de esta lengua a nivel de Educación Inicial, aunque la iniciativa no cubre todas las escuelas del país. No obstante, este par de hechos llaman la atención sobre la importancia social y económica que el inglés venía teniendo en las clases medias y el sector público, pero no debemos olvidar que desde inicios de esta misma década las clases medias altas estaban propiciando una movilización importante de las instituciones de educación pública a la privada.

Esta movilización se da a partir del desencanto general con respecto a las calidades del sector educativo público y que este no podía resolver. Las clases económicamente más solventes pueden cubrir el costo de una educación privada que les promete calidad. El punto de tensión saltó a partir de la enseñanza de la computación y del inglés como elementos deseables de la educación con miras a la preparación para el mercado laboral. Es desde esta óptica que se instauran ambas materias como saberes deseables para cumplir con las nuevas demandas laborales de un mercado cada vez más delimitado por la IED y que la sociedad reclama a la educación como símbolos de calidad. A partir de esto las empresas turísticas y las de servicios al cliente vendrían a consumir la mano de obra preparada en el

\footnotetext{
${ }^{2}$ Ver Córdoba, Coto y Ramírez (2005, p. 3) para identificar ejemplos claros de las múltiples instituciones de enseñanza media en donde se empiezan a incorporar el inglés como materia de estudio obligatoria.
} 
uso de las computadoras y del inglés, al mismo tiempo que pagaría mejores salarios que los asequibles en los campos tradicionales.

Es en este marco que, para el caso, aprender inglés asume un nuevo significado social y simbólico. Ya para finales de la década de los noventa saber hablar inglés se asocia con la consecución de una plaza laboral mejor pagada, salarios en dólares y en campos menos tradicionales que los acostumbrados hasta el momento por la gran mayoría de costarricenses de clases medias.

Más recientemente, y acorde con el discurso histórico, económico y educativo sobre la importancia del inglés (especialmente el referido a la clase media) se desarrolló el Plan Nacional de Inglés (2007). Este plan fue elaborado por el Ministerio de Comercio Exterior (COMEX), el Ministerio de Economía, Industrias y Comercio (MEIC), el Ministerio de Educación Pública (MEP), el Instituto Nacional de Aprendizaje (INA), CINDE (Coalición Costarricense de Iniciativas de Desarrollo) y el proyecto Estrategia Siglo XXI (Plan Nacional Costa Rica Multilingüe). El Plan Nacional de Inglés señala que

La inserción de Costa Rica en los mercados internacionales ha propiciado la diversificación de las exportaciones y el aumento de la inversión extranjera directa (IED) en sectores no tradicionales (servicios de alta tecnología, dispositivos médicos y electrónica) además del sector turístico. Sin duda, el país ha tenido relativo éxito en su posicionamiento global... Por ello, las condiciones actuales de competitividad país llevan a Costa Rica a plantearse diversos retos, uno de estos es específicamente el desarrollo de las competencias lingüísticas en un segundo idioma que para todos los efectos tiene como prioridad mundial el inglés... Lo anterior es evidente en la demanda de recursos humanos con formación bilingüe, por parte del sector productivo nacional y transnacional. $(2007$, p. 2)

Al respecto, el CINDE (2010, p. 4) también argumenta que el Plan Nacional de Inglés "responde a la necesidad del país de cumplir con la creciente demanda por parte del sector productivo, de capital humano con dominio del idioma Inglés". Desde la perspectiva empresarial, pareciera que el sistema educativo público es el "semillero" de la mano de obra de sus empresas y de las transnacionales. Es decir, no está mal que las personas tengan la capacidad de incorporarse al mercado laboral justo cuando terminan la secundaria o antes si es posible, pero pensar que esta es la única opción que tiene el país y las personas que 
estudian en el sistema educativo público, es una visión muy pobre de la sociedad, de la vida y del sistema productivo mismo.

Así, se puede deducir del discurso "desarrollista" que todo el aparato educativo está en función del aparato productivo-económico, pues se tiende a ver la relación histórica existente entre educación y desarrollo únicamente en esos términos. Al respecto el CINDE (2010, p.2), también apunta que

Desde 1870, la escuela primaria ha sido gratuita y obligatoria. Esto ha establecido las bases para una mejora continua en la educación universitaria y la capacitación técnica, que a su vez ha mejorado el ambiente empresarial y ha atraído compañías extranjeras para que se establezcan en Costa Rica.

Es bastante obvio que la educación, y dentro de ella la enseñanza del inglés, se perfila dentro de la lógica de preparación de mano de obra calificada, esto para que las corporaciones transnacionales y las pequeñas y medianas empresas nacionales puedan disponer de abundante mano de obra preparada, con el fin de que les permitan globalizar sus negocios, situación que a simple vista no pareciera dañar a nadie, pues lo único que se desprende de estas citas refiere a la abundancia de trabajo, situación más que deseable en momentos como los actuales para la economía de Costa Rica.

También es bastante obvio que el tema educación, tal y como es referido en esas propuestas, se adhiere a un discurso que entiende y dispone del sistema educativo público dentro de un continuum de organización socioeconómico que no debe alterarse y dentro del cual el tránsito de individuos de un estrato a otro es producto del esfuerzo individual y no del colectivo. Es evidente que los colegios públicos deben dedicarse a la preparación técnica de la mano de obra calificada y por ello la enseñanza debe limitarse a este espacio. El acceso a los conocimientos científicos como la robótica o las nano y biotecnologías son espacios reservados para las clases más altas o las instituciones con "mejores estudiantes" (Colegios Científicos), así como la educación donde se perfilen otras visiones de mundo, talvez no tan concordantes con el "desarrollismo", son ajenas a las políticas culturales que emanan de los documentos citados anteriormente, al menos para las instituciones donde se concretan estas políticas y propuestas: los colegios públicos pobres.

Nuevamente salta la disyuntiva de si esta no es la mejor alternativa para la educación de los grupos económicamente menos solventes, pues es mejor un empleo que la tradicional 
mala educación que se describe en el Primer Informe Estado de la Educación. Tal parece que para la mayoría de familias de estudiantes de esas instituciones no existe mejor alternativa o que al menos hasta este momento es lo menos malo que la educación pública les puede ofrecer.

Desde la academia parece existir un consenso con las políticas económicas y educativas en lo referente a la necesidad e importancia del inglés. Aunque no podemos interpretar la voluntad de la totalidad de individuos, la forma en que se justifica el quehacer, en los textos que aquí se presentan, se convierte en discurso social y es aprobado, no solamente por el grupo de especialistas sino por las comisiones de expertos en pares ciegos y comités editoriales, situaciones que permiten entender la aceptación de tales propuestas. Así, Hernández (2008, p. 2) justifica la enseñanza y aprendizaje del inglés en virtud de que

Costa Rica es un país que se encuentra muy abierto al comercio internacional, por lo que existen muchas empresas nacionales e internacionales que requieren personal con dominio de una segunda lengua. La prioridad mundial en este momento es el inglés ya que éste se ha convertido en el "segundo idioma del mundo". Las personas de diferentes nacionalidades generalmente utilizan esta lengua para comunicarse entre sí, de manera que la habilidad para hablarlo, leerlo y escribirlo se ha convertido en un requisito indispensable para conseguir un buen trabajo... Obviamente Costa Rica necesita mejorar la enseñanza del inglés. Una de las formas de hacerlo es que las instituciones superiores preparen personas aptas para diseñar cursos de inglés con Propósitos Específicos (ESP), con el fin de capacitar al estudiantado para desempeñar tareas específicas en su situación laboral utilizando el inglés. De esta forma, se podrá solventar a corto plazo la demanda insatisfecha de los sectores empleadores de personal con dominio de una segunda lengua.

De igual forma, Navarro y Peñeiro (2009, pp.130-131) indican que

El idioma inglés ha desarrollado un carácter de lengua franca debido al uso alcanzado en el mundo internacional de los negocios. En un ámbito de relaciones comerciales, no solo es considerado el idioma más utilizado, sino también el más hablado. Esta particularidad indica la importancia de que los costarricenses cuenten con el inglés como herramienta de comunicación, que les permita alcanzar niveles de competencia lingüística en varios ámbitos... A raíz de estas propuestas curriculares, promovidas por 
las autoridades educativas del país, cuyo objetivo es fortalecer el aprendizaje del inglés y otras lenguas, la educación pública superior debe a la vez considerar su papel en este proceso, para responder a las exigencias del mercado laboral en relación con la formación de profesionales bilingües (español-inglés) y mantenerse a la vanguardia como ha sido su característica en la historia costarricense.

Álvarez y Bassi (2010, ๆ 2) también consideran que

Dominar el inglés como segunda lengua será una habilidad indispensable para acceder a mejores oportunidades en este nuevo siglo. La lengua inglesa es la más útil para la comunicación internacional, interregional y multilateral y es la lengua franca en ámbitos como el mercadeo, el comercio internacional y el turismo. Además, es el idioma de curso corriente en la academia y las ciencias, y la principal puerta de acceso a la tecnología de punta.

Para una gran mayoría de docentes está claro que para atraer la inversión extranjera, los gobiernos de turno han promovido una política económica y educativa en donde la enseñanza y el aprendizaje el inglés son clave. Es en este sentido que realizan sus propuestas académicas y que visualizan sus quehaceres dentro de las carreras y cursos de inglés.

Lo que parece no estar decodificado, es que en este contexto histórico, políticoeconómico y educativo planteado, las personas son recursos humanos a merced de mercado y por ende de la economía transnacional. Dicho de otro modo, pareciera que los hablantes costarricenses del inglés se visualizan como sujetos y objetos de competitividad mercantil en donde la vida digna está siendo medida a través de estándares de pobreza que como se explicará a continuación son muy cuestionables, y donde las alternativas desde la educación parecen ser un lujo costoso e improductivo para la sociedad.

\section{Enseñanza y aprendizaje del inglés como mercancía y su impacto en la pobreza}

El resultado del modelo económico escogido ha propiciado la construcción de una identidad socio-educativa en la enseñanza y aprendizaje del inglés basada en la noción de empleo, y por ende, en la asociación metonímica con el estado económico de las personas. En un estudio etnográfico sobre el saber docente, Córdoba y Araya (2010, p. 34) explican 
que uno de los principios epistemológicos y axiológicos más fuertemente identificados en un grupo de profesores de la enseñanza del inglés está basado en

que el inglés es necesario por la única razón de que las personas hablantes del inglés tienen la oportunidad de acceder a más bienes y servicios pero desde la posición de empleado. En este sentido, los horizontes laborales se dibujan en las empresas; principalmente trasnacionales, de atención al cliente que comercializan bienes y servicios. El otro horizonte es el turismo donde a través del inglés se espera conseguir trabajos de recepcionistas, secretarias, meseros o como guías de turismo para alguna agencia de tours operadores, entre otros. Esta noción económico-funcional de la lengua inglesa es la principal justificante para su enseñanza-aprendizaje, porque el objetivo principal de las autoridades gubernamentales -situación también asumida desde las carreras- es generar mano de obra calificada al servicio de las compañías y corporaciones trasnacionales.

Así, las personas han ido creando - sin percatarse sobre los principios capitalistas de costo y demanda - imaginarios económicos en los que la enseñanza y aprendizaje del inglés figuran como medios para salir de la pobreza. El inglés, entonces, se ha venido a instaurar en la consciencia colectiva de las familias, estudiantes, profesorado y autoridades políticas como un bien y un servicio; mercancía y objeto que permite el consumo. Pero para abordar el tema de la enseñanza y aprendizaje del inglés como mercancía resulta imperante hacer algunas aclaraciones conceptuales de lo que el término pobreza significa desde diferentes dimensiones.

Popularmente, el concepto de pobreza ha sido relacionado con el estado socioeconómico que los individuos poseen a la luz de los modelos capitalistas y sus posibilidades de consumo dentro de ese paradigma económico. Desde tal perspectiva, Baratz y Grigsby (1971, p. 120, en Spicker, 2009, p. 292) definen la pobreza como "una privación severa de bienestar físico y bienestar mental, estrechamente asociada con inadecuados recursos económicos y consumos". Esta relación entre bienestar y acceso económico genera de acuerdo con Trejos (1990, p. 3) "la presencia de niveles de vida o bienestar socialmente inaceptables".

Al respecto, Spicker (2009) señala la existencia de varios conceptos de pobreza (al menos doce aborda el autor) dentro de los cuales la pobreza como concepto material es una 
de las ideas más comunes. En este sentido, Spicker (2009, p.292) apunta que "[e]/ primer grupo de definiciones se refiere a la pobreza como un concepto material. La población es pobre porque no tiene algo que necesita, o cuando carece de los recursos para acceder a las cosas que necesita". Estas definiciones son congruentes con posiciones ideológicas emanadas de instancias internacionales, como el Banco Mundial o el FMI y se proyectan como enfoques dentro de los cuales toman sentido esas definiciones y se enmarcan las políticas de los países. Para este autor "[u]no de los enfoques más utilizados para medir la pobreza es el de los ingresos, a tal punto que algunos científicos sociales consideran que pobreza es equivalente a bajos ingresos." (Spicker, 2009, p. 294). Por esta razón, explica Hopenhayn (s.f., p. 2)

la forma consagrada y convencional de medir la pobreza está correlacionada estrechamente con la disponibilidad o no de ingresos para satisfacer necesidades básicas. La ecuación ha consistido en estimar el costo de una canasta familiar y cotejarlo con los ingresos de las familias. La combinación de cálculo económico con información censal y de encuestas de hogares permite ir corrigiendo datos que nos hablan del porcentaje de pobres en un país cualquiera. Así, se ha definido como pobre el grupo familiar cuyos ingresos son inferiores al doble del monto fijado para adquirir mensualmente una canasta familiar, y como pobre extremo a aquellas familias cuyo ingreso mensual no supera el precio de una canasta familiar.

Esta fórmula es la que se ha utilizado en Costa Rica para determinar el nivel de pobreza nacional.

Con relación a la enseñanza y aprendizaje del inglés, como ya se ha evidenciado anteriormente, su necesidad e importancia han radicado históricamente en el valor agregado que representa esta lengua para la economía transnacional a través de una mano de obra que espera ser contratada. En este panorama se sostiene que enseñar y aprender inglés va a proporcionar mayor atracción de mercados extranjeros y por ende mayor inversión local que se traduciría en más empleos y consecuentemente en un mejoramiento en los niveles económicos de los hablantes. Pero en estás relaciones comerciales y laborales, enseñanza y aprendizaje del inglés versus pobreza resulta imperativo aclarar la filosofía que sustenta los principios de una economía transnacional - y si se quiere nacional - de orden capitalista. 
En términos muy generales, para lograr un nivel deseado de ganancias las corporaciones han demandado de abundante mano de obra técnica-especializada que hable inglés y sepa usar computadoras. Pero no se ha tomado en cuenta que en un sistema capitalista, el exceso (en este caso de mano de obra calificada) permite abaratar costos de operación pues la competencia laboral mantiene un dominio tácito sobre los salarios que se les pagan a los empleados. A pesar de que se ha querido promover la idea de que la enseñanza y aprendizaje del inglés figuran como indicadores estratégicamente clave para el mejoramiento económico de las familias, no existe ningún estudio científico que asocie el manejo de esta lengua con variables que reflejen un mayor ingreso económico y si este se da realmente por el uso de esa lengua. No obstante en Costa Rica esta lógica capitalista es la que ha dominado el tema de la pobreza, especialmente en los sectores educativos y gubernamentales. En consecuencia, generar un empleo se ha convertido en la alternativa exclusiva para combatir esos niveles de vida inaceptables.

En este sentido, resulta más bien contradictorio ver que mientras la inversión y puesta en práctica de políticas que estimulen e incrementen el dominio del inglés han sido significativamente cuantiosas (lo cual supone mayores hablantes actualmente con relación a los existentes anteriormente), los índices de pobreza en Costa Rica no han disminuido dramáticamente. Contrariamente, por ejemplo, Jiménez, Céspedes y Arce (2006) identifican dos períodos histórico-económicos relevantes con relación a la pobreza familiar. Los autores afirman que de 1987 a 1990 el número de familias pobres disminuyó poco pero la pobreza repuntó de 1990 a 1991; de 1991 a 1994 las familias pobres sí disminuyeron considerablemente pero de 1994 al 2004 el estancamiento económico más allá de la disminución de la pobreza ha sido inminente, situación que contrasta con el creciente número de personas que egresaron de instituciones privadas de tipo "bilingüe", es decir, que para ese decenio las personas hablantes de inglés se habrían multiplicado en todo el territorio nacional, pero la situación económica fue evidentemente desfavorable para todo el país.

El siguiente gráfico presentado por Jiménez, Céspedes y Arce (2006, p. 60) ilustra mejor la forma en que la pobreza económica entre familias costarricenses ha evolucionado en los años de 1987 a 2004: 


\section{Grafico 1}

PORCENTAJE DE FAMILIAS POBRES, 1987-2004

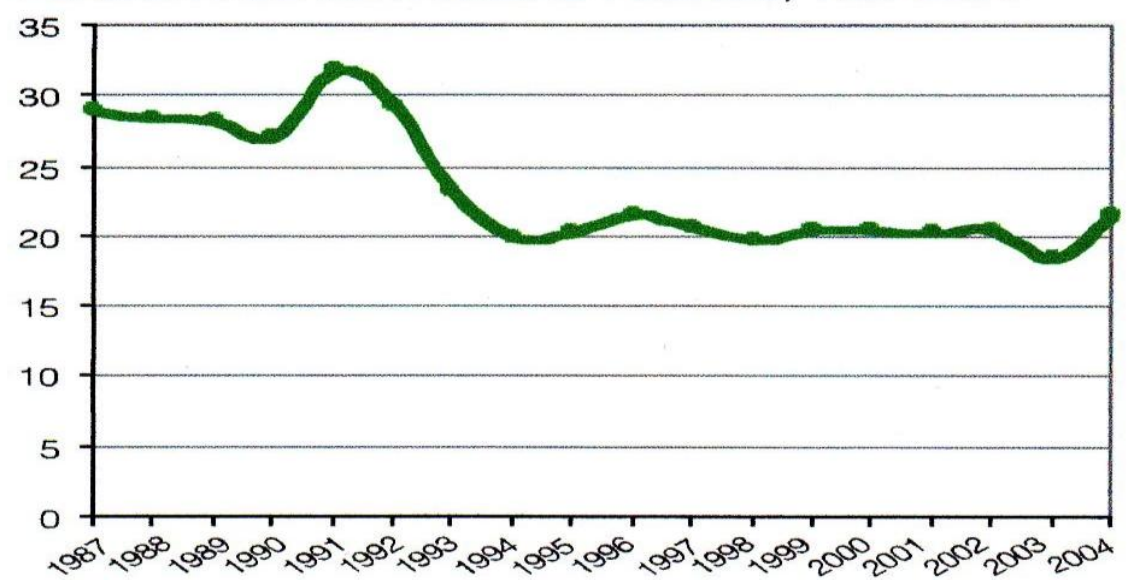

FUENTE: INEC, Encuesta de Hogares de Propósitos Múltiples.

En el siguiente cuadro presentado en el documento Políticas públicas regionales sobre la reducción de la pobreza en Centroamérica y su incidencia en el pleno disfrute de los derechos humanos: informe nacional Costa Rica (Consejo Centroamericano de Procuradores de Derechos Humanos, 2008, p. 51) también se puede observar como los niveles de pobreza se han mantenido casi invariables en el período del 2004 al 2006:

Tabla 1

\begin{tabular}{|c|c|c|c|c|}
\hline \multicolumn{5}{|c|}{ Porcentaje de hogares pobres en Costa Rica 2000 - 2006} \\
\hline Año & $\begin{array}{c}\text { No } \\
\text { pobres }\end{array}$ & Total & $\begin{array}{c}\text { Pobres no satisface } \\
\text { necesidades básicas }\end{array}$ & $\begin{array}{c}\text { Extrema } \\
\text { pobreza }\end{array}$ \\
\hline $2000^{\star}$ & 79,4 & 20,6 & 14,5 & 6,1 \\
\hline 2001 & 79,7 & 20,3 & 14,4 & 5,9 \\
\hline 2002 & 79,4 & 20,6 & 14,9 & 5,7 \\
\hline 2003 & 81,5 & 18,5 & 13,4 & 5,1 \\
\hline $\mathbf{2 0 0 4}$ & $\mathbf{7 8 , 3}$ & $\mathbf{2 1 , 7}$ & $\mathbf{1 6 , 1}$ & $\mathbf{5 , 6}$ \\
\hline 2005 & 78,8 & 21,2 & 15,6 & 5,6 \\
\hline 2006 & 79,8 & 20,2 & 14,9 & 5,3 \\
\hline
\end{tabular}

*Datos recalculados con el Censo del 2000, no mediante la Encuesta de Hogares con Propósitos Múltiples Fuente: Rojas Benavides, Carla. A partir de datos proporcionados por el INEC, 2007. (Resaltado original del texto). 
Pero la realidad histórica de la pobreza nacional sólo refleja una tarea no resuelta en el campo económico.

En la óptica del desarrollo humano, la pobreza es más amplia que la falta de ingresos y en ese sentido, junto a la pobreza de ingresos, se describe la pobreza humana, [...] la falta de posibilidades para desarrollar plenamente la capacidad de las personas. (Artigas, 2003, en IIDH, 2008, p. 21)

Al respecto, como bien lo expone Nayaran, "la pobreza nunca se produce debido a la falta de un solo elemento, sino que es consecuencia de múltiples factores relacionados entre sí que inciden en las experiencias de la gente y sus definiciones de la pobreza" (2000, p.32). Así que la definición tradicional de pobreza como estado material-económico resulta ser insuficiente y sumamente materialista, especialmente si uno considera, entre otras, las realidades y problemáticas psicosociales, ecológicas y culturales de los individuos como seres sociales en su calidad humana y no material. Sobre este punto cabe cuestionar, entonces, la importancia histórica que se le ha asignado a la enseñanza y aprendizaje del inglés puesto que desde la dimensión del impacto económico en las familias costarricenses no el de las empresas - el inglés no puede ser visto como un fin en sí mismo que implique automáticamente un mejor nivel socioeconómico. Si la pobreza es económica, al menos debería de existir una política -primeramente - de mayor cobertura universitaria pues como lo indica el siguiente gráfico de Jiménez, Céspedes y Arce (2006), la tendencia hacia ser económicamente pobre aumenta o disminuye según el grado educativo de la población:

\section{Gráfico 2}

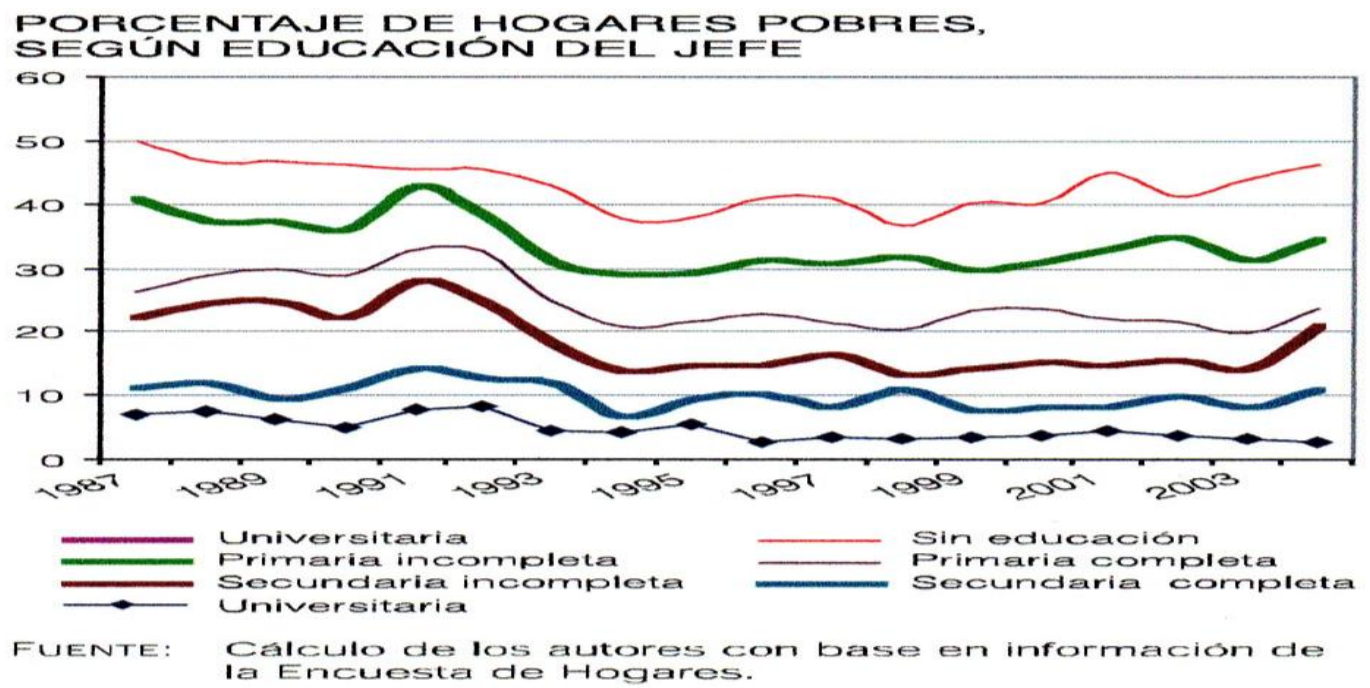


Ante estos datos es necesario preguntar si una persona que sepa hablar inglés pero no tiene educación primaria o secundaria completa (situación que no se aleja de la realidad costarricense si uno considera 1, los altos porcentajes de deserción estudiantil y 2, la política e inversión en educación técnica), podrá acceder a buenos empleos con salarios dignos que faciliten el mejoramiento de su situación socioeconómica. O si por el contrario, la diferencia de su nivel socioeconómico radica más bien en el acceso que ese individuo pueda tener a una educación universitaria de calidad académica y humanista en la que la enseñanza y aprendizaje del inglés figure como un medio de acceso y difusión del conocimiento a nivel nacional e internacional.

Es indiscutible que es mejor tener un empleo que no tenerlo, y ante la desesperante situación de pobreza que enfrentan las familias costarricenses pobres, generar un empleo se ha convertido en la alternativa exclusiva para combatir los niveles de pobreza, muchas veces sin medir ciertas consecuencias, porque, como se ha visto en este tiempo, para algunos sectores es socialmente aceptable generar y acceder a un empleo para "salir de pobre", muy a pesar de que esto signifique la destrucción de los ecosistemas o la contaminación del agua que ingiere la propia familia y la comunidad del mismo trabajador.

\section{Algunas repercusiones del concepto de pobreza material}

La noción de pobreza material se inscribe en un enfoque economicista re-productor de ideas y prácticas que tienden a descontextualizar las relaciones objetivas y subjetivas entre los individuos y sus bases materiales invisibilizando la fuerza de la cultura y los estados de poder así como el dominio al que son sometidos los individuos. En este sentido Romero asegura que "la pobreza no se expresa sólo en la ausencia de bienes materiales de consumo, las actitudes conformistas producen una miseria aun más grave" (1996, p. 13). Para Romero existen otros tipos de pobreza más allá de lo meramente económico y consumista, situación que agrega relevancia al fin de este trabajo. No obstante, Romero le adjudica al conformismo la génesis de esos otros tipos de pobreza cuando en realidad es en sí una manifestación de una pobreza específica. Por otra parte, quizás más significativamente, la definición de Romero así como muchas semejantes, desconoce las condiciones históricas y socioculturales en las que se generan las necesidades pero también los conocimientos que le permiten a un individuo reinterpretar su contexto y actuar en y sobre éste. 
Al mismo tiempo, el tema de la pobreza económica refleja un fetiche político mitificado en discursos deterministas y politiqueros al servicio del poder y la dominación. Es decir, la pobreza en la medida que exista reducida sólo al ámbito económico, y que no se perciba como tal en otros escenarios como el ambiental, espiritual o cultural, es capaz de desviar la atención de importantes sectores sociales y sacar de sus agendas temas de gran trascendencia nacional. Uno de los mejores ejemplos de esta deslocalización de atención se encuentra en el marco de lo que fue la votación del referéndum del Tratado de Libre Comercio (TLC) entre Centro América y los Estados Unidos donde se evidenció que el Sí ganó terreno bajo la premisa de mejores salarios, mayor variedad de productos, abaratamiento de precios y tarifas y del miedo a perder empleos. Mientras que las tesis del No, que abogaban por temas críticos y urgentes en ámbitos como la soberanía, la identidad, la propiedad intelectual y el medio ambiente, más allá del empleo y del consumismo, no pudo revertir, aunque por poco, el impacto de las anteriores. Incluso sectores académicos de las universidades públicas manifestaron su apoyo al TLC aun cuando éste claramente atenta contra el modelo en que se sostiene la universidad pública.

A diferencia de los planteamientos anteriores, encontramos en Pérez, Mora y Morales una muy interesante propuesta para operacionalizar el concepto pobreza, donde se develan diferentes formas de poder y una posible relación de la pobreza con nuestro quehacer académico. Para ellos

[l]a sociedad en América Latina tiende actualmente a ser visualizada en términos de niveles de pobreza e integración social... Esta visión predominante ha sido impuesta, fundamentalmente, por organismos internacionales que han colonizado el imaginario sobre la sociedad y han impuesto una mirada seudo-crítica sobre la realidad. Así, en primera instancia aparece como denunciadora de carencias sociales, pero su comprensión es mixtificadora porque no expresa relaciones sociales antagónicas basadas en el poder. (2007, p. 3)

Es evidente que la pobreza, expresada en esos términos de integración y carencias, no permite entender diferentes formas de articulación de la educación con la economía, sino que reduce esta relación a una causalidad unidireccional: mayor/menor educación es igual a mayor/menor posibilidad de un mejor empleo.

A la luz de todas las problemáticas del siglo XXI, es necesario re-plantearnos la 
enseñanza y el aprendizaje del inglés como actividades humanas concretas que transitan entre espacios subjetivos y objetivos de la mente del individuo y pueden enriquecer o empobrecer bagajes culturales, entendimientos, percepciones y aptitudes. Es en este marco que Medrano, Varillas y Bonilla (2002, pp. 30-31), aciertan al decir que

El paradigma del desarrollo a escala humana nos plantea que hablar de pobreza es un reduccionismo economicista, lo que hay son pobrezas porque cualquier necesidad humana fundamental que no es adecuadamente satisfecha, revela pobreza específica. Desde esta perspectiva, se puede señalar que en Centroamérica se encuentra la pobreza de subsistencia, con altos índices de población con abrigo y alimentación insuficiente; pobreza de protección, con las diferentes expresiones de violencia, inseguridad ciudadana y sistemas de salud ineficientes; pobreza de afecto, con la prevalencia de relaciones interpersonales y estilos de liderazgo autoritarios; pobreza de entendimiento, con los altos índices de analfabetismo y escasas oportunidades de acceso a avances tecnológicos; pobreza de participación, debido a la marginación y discriminación de mujeres, niñez, adolescencia, juventud e indígenas; pobreza de identidad con el culto al consumismo... (el subrayado es mío)

En el contexto educativo de la enseñanza y aprendizaje del inglés con relación al enfoque de pobreza material, es necesario reflexionar sobre los discursos y saberes que se legitiman o marginalizan dentro de los programas de educación superior. Esto por cuando en última instancia es en la universidad a través de los bachilleratos, licenciaturas y maestrías en la enseñanza del inglés que se re-producen posiciones ideológicas y se desarrollan aptitudes y actitudes en el estudiantado que en futuro será la fuerza encargada de llevar a cabo las políticas educativas del país. Re-pensar la enseñanza y aprendizaje del inglés a la luz de otros tipos de pobrezas como las ambientales, culturales, morales y espirituales demandaría una reflexión profunda sobre el por qué para la mayoría de autoridades educativas (entre ellas la universitaria), profesores y población en general, la importancia del inglés no se enmarca en su poder ideológico-discursivo como legua que es; en el poder que le asiste como medio de comunicación para construir y re-deconstruir visiones de mundo que sean respetuosas de la dignidad humana y del medio ambiente. En lo concreto, la reflexión también requiere una evaluación sobre los contenidos, objetivos, metodologías de los cursos, los tipos de cursos que se ofrecen a la población estudiantil, los perfiles profesionales 
de salida, las habilidades que se privilegian y castigan en las evaluaciones para así determinar las pobrezas adyacentes nacidas del discurso economicista que ha marcado la identidad de los procesos de enseñanza y aprendizaje del inglés, principalmente, para la clase media y pobre costarricense.

\section{Las pobrezas y la enseñanza y aprendizaje del inglés}

El planteamiento hecho desde la pobreza material y sus implicaciones sobre las necesidades humanas fundamentales así como sobre cuestiones éticas, espirituales, ambientales y culturales, permite re-pensar el trabajo desde una perspectiva más amplia a la de la consecución de un salario. En un sentido más holístico y humano, la razón de ser de los programas en enseñanza y aprendizaje del inglés requieren un abordaje más profundo de lo que hasta ahora han revelado algunos estudios sobre la construcción del saber docente; saber tradicional centrado en la especialidad, en lo técnico, en lo legaladministrativo o en la idea de combatir la pobreza al posibilitar la consecución de un trabajo (Ver Araya y Córdoba, 2008 y 2010).

Al aplicar un concepto amplio de pobrezas que permita categorizar las necesidades humanas fundamentales en el análisis de los diferentes quehaceres de los planes de estudio en enseñanza y aprendizaje del inglés, sobresale inmediatamente el hecho de que la enseñanza y aprendizaje del inglés no puede ser una disciplina que refiera a una especialidad desvinculada y despreocupada de los discursos que re-produce sobre otros saberes hasta ahora no propios del saber docente.

En este sentido, desde el ámbito académico, la acción social e investigación en la enseñanza y aprendizaje del inglés es necesario asumir la objetivación y subjetivación de las pobrezas como un estado humano que transciende lo económico. Al respecto, se plantean aquí, y a modo de ejemplo, una serie de instancias relacionadas con algunos tipos de pobreza que se re-producen cuando se considera que el inglés es importante y necesario sólo para encontrar un empleo.

\section{Pobreza de identidad}

En una primera instancia pareciera que la pobreza de identidad estaría muy estrechamente relacionada con todo el quehacer y política cultural generados desde y de la que es objeto la academia. Por tal razón es imperativo revisar las creencias en cuanto a lo 
que significa ser costarricense-centroamericano-humano y hablar inglés. Esta lengua llega a enmarcase en las culturas nacionales ¿podrá empobrecerlas?, ¿tendrá la capacidad de aportar elementos que enriquezcan las culturas locales? Desde los procesos de aula, de la investigación y de la acción social en la enseñanza y aprendizaje del inglés ¿qué elementos se pueden incluir para afirmar las identidades locales y cuáles deben ser motivo de revisión para entender y actuar sobre sus efectos no compatibles con los principios que dictan las Políticas Universitarias?

Un insumo importante para la discusión sobre las interrogantes planteas se encuentra en el trabajo de Spence (2004) quien advierte de las pobres relaciones que tiene el trabajo académico en la afirmación y legitimación de las culturas y la diversidad lingüística que caracterizan a Costa Rica y los pueblos y etnias que aquí conviven. Según la autora el "criollo limonense" está siendo menos usado por la población de hablantes menores de 21 años de esa provincia. Desde las universidades, entonces, ¿están los programas de estudio de la lengua inglesa contribuyendo con este tipo de pobreza identitaria?, ¿podrán las diferentes carreras aportar elementos para la reafirmación y legitimación de las identidades en nuestras culturas?, ¿podrían los docentes algún día y de alguna manera contribuir para que el inglés de Limón sea valorado y apreciado en cuanto lengua inglesa propia costarricense que enriquece nuestro bagaje cultural mucho más allá de la exuberancia o la trivialidad del libro de texto?

A simple vista se sabe que la respuesta no es fácil. Es decir, identificar los elementos enriquecedores y los que no lo son, implica un arduo trabajo que va más allá de un listado de cosas, implica tomar una posición en tiempo y espacio con respecto al objeto sociedad, lengua y cultura desde el cual se pueda iniciar una negociación de significados con cada sujeto en las carreras en la enseñanza del inglés. Esta negociación debería resultar en la construcción y aplicación de herramientas teórico-conceptuales con las que cada estudiantedocente pueda visualizar, entender y resignificar la lengua inglesa, su aprendizaje y su enseñanza bajo una actitud respetuosa y crítica de lo que lingüística y culturalmente se ha marginalizado. El impacto de semejante crítica debe reflejarse en las temáticas, en los contenidos, en las herramientas y los propósitos de la enseñanza y el aprendizaje del inglés, además de los objetivos y criterios de evaluación de los cursos. Para ello es necesario entonces una clara posición con respecto al papel de la lengua inglesa en nuestra cultura más allá de la consecución de una plaza laboral. 
Dentro de estos límites se pueden apuntar un par de ejemplos más a mano y más representativos de esos saberes antidemocráticos encarnados en dos saberes técnicos propios de la enseñanza del inglés: enseñar la variable estándar y la utilización de libros de texto.

La situación con dicha variable, existe un gran consenso en esto, es que se entiende como la más accesible o entendible para la gran mayoría de personas hablantes nativas y no nativas de esa lengua. Sin embargo, como lo apunta Jenkins (2006, p. 32) "[l]a falta de aceptación de las variables nativas no estándar tienen una aparente conexión con actitudes racistas en USA y clasistas en UK" (traducción mía), situación que viene a plantear un cuestionamiento ético y moral a la enseñanza de la variable estándar que se piensa se enseña en el Bachillerato, ya que, por una parte, estas instancias racistas y clasistas no deben, bajo ninguna óptica, ser reproducidas en nuestra universidad. Por otra parte, la gran mayoría de docentes quienes laboramos para esta carrera no somos nativos del idioma y tendemos a resolver el asunto de la variabilidad lingüística dentro del marco de los libros de texto y los diccionarios; es decir, son estos materiales los que demarcan lo que es y lo que no es estándar, pero un libro o un material utilizado de forma indiscriminada y prescriptiva siempre deja mucho del mundo real por fuera.

No obstante, lo que se propone como variable estándar de la lengua inglesa en la gran mayoría de textos lingüísticos, y lo que se articula como saber en la enseñanza del inglés son, generalmente dos cosas muy diferentes. La variable estándar, parafraseando a Carr (1999), puede ser utilizada en términos de la sintáctica y el vocabulario de esta variable, mientras se mantiene el acento - la fonética y la fonología - de la lengua nativa. Por su parte, Crystal (2004) propone que es a partir del contacto internacional que las diferencias entre dialectos se acortan y que las tecnologías de la información y la comunicación tienen un gran peso en la adquisición y aprendizaje del vocabulario "estándar" y en la estandarización de vocablos no entendidos como tales anteriormente, así como también son un gran medio para el conocimiento de las fonéticas y fonologías de otras variables 0 dialectos.

Sin embargo, lo que se entiende por variable estándar en muchos cursos del Bachillerato dista enormemente de lo que se propone en esos textos lingüísticos. La gran mayoría del trabajo de los cursos de la carrera se han hundido en lo que Fairclaugh (2001, p. 6) ha llamado "una visión idealizada de la lengua" (traducción mía), en la que esta no cambia 
ni en tiempo ni en espacio. Esta situación fomenta la predilección por aquella variable estándar que viene en los libros y termina desestimando y desvalorizando otras variables también estándar - del inglés hablado también como lengua materna en Costa Rica. Lo que se entiende como bueno-malo, deseable-no deseable se reduce al manejo del vocabulario de los libros de texto y a la capacidad de articular los contenidos en oraciones sintáctica y fonéticamente correctas, como que si esto representara la única forma de hablar un inglés de prestigio.

Existe toda una situación que se encierra en las páginas de los libros de texto que no debe ser inadvertida , ya que como apunta Jenkins (2000, p.14),

la mercadotécnia de libros de textos para la enseñanza del inglés como lengua extranjera (EFL) se ha investido con la autoridad de mantener el estatus quo de la fonología, dando gran énfasis a una supuesta necesidad de que las personas que aprenden inglés en esta modalidad reproduzcan características de la pronunciación propias de 'hablantes nativos', (sea del RP o del General American), aun si estas resultan irrelevantes para la inteligibilidad del discurso. (Traducción mía)

Se podrían entender los párrafos anteriores como algunas de las tareas - si existe algún acuerdo - que son urgentes, desde lo que se entiende como una variable o dialecto deseable y a enseñar, hasta lo que entendemos como un acto común y consecuente con la labor docente: escoger un libro de texto. Estas acciones, si se revisan detenidamente, pueden significarse como pobrezas de identidad, donde la lengua inglesa se consume como un bien que da estatus y donde se puede acceder al reducto último de la lengua inglesa pagando por este: el libro de texto. Así, se convierten en elementos más importantes los materiales y el estatus que los contenidos, el desarrollo de la criticidad o de las sensibilidades a las variaciones lingüísticas, para anotar solamente algunas. Por esto es que se aprecia más deseable sentarse a platicar inglés con alguien que viene de los Estados Unidos que con un nativo hablante del inglés de Limón, ya que esta lengua no se encuentra en ningún libro de texto para la enseñanza del inglés sino en la vida real, pero en un contexto que no genera un deseo de consumo ni es posibilitador de algún estatus deseable.

Por otra parte, la población estudiantil, en términos generales, llega a las aulas visualizando su paso por la universidad como un medio para satisfacer sus necesidades de consumo. Su fin más mediato es la consecución de un título que se traduzca en un empleo 
bien remunerado. Esto no es malo. Sin embargo, en las carreras de las universidades públicas se debe tener la capacidad para aportar elementos, desde la misma lengua inglesa, con los que cualquier individuo pueda resignificar su vida a partir de parámetros más amplios que los del consumismo, anteriormente apuntamos dos.

Si existe un consenso entre autores sobre la esencia de la globalización es su exacerbado énfasis en el consumismo lo cual es característico de la pobreza de identidad. Así el discurso de trabajo-salario vs. pobreza-desempleo es complaciente no solo con los fines neoliberales de acaparar las instancias de poder, sino que también imposibilita la valoración de nuestras propias identidades, ya sea por asumir actitudes complacientes, cortoplacistas y poco críticas, o debido a que el consumismo se convierte en un fin en sí mismo en donde la enseñanza y aprendizaje figuran como un medio para lograr su propósito.

Al respecto, la propaganda neoliberal ha vendido el sueño del progreso a partir de la instalación de empresas turísticas y transnacionales en suelo nacional para las cuales uno de los primeros requisitos como ya se ha dicho es hablar inglés. Desde la academia se vive ese sueño de una forma muy diferente a como la viven los jóvenes de Jacó (Cfr. programa Siete Días, Canal 7 de CR, lunes 8 de septiembre de 2008). En términos generales, las personas que aprenden inglés creen en la abundancia de trabajo a partir del aprendizaje de esta lengua. Sin embargo, la exclusión social y económica es la realidad de la población joven de Garabito, quienes ven un futuro muy diferente al que se les ofrece en la propaganda estatal y corporativa de muchas de esas empresas que se pretenden ofrezcan buenas oportunidades laborales.

La concentración de tierra en manos de unas cuantas compañías extranjeras, la prostitución, la drogadicción y las relaciones laborales insanas son espacios entre los que la población joven tiene que darle sentido a sus vidas y construir sus sueños, sus deseos y su futuro. En este punto es oportuno preguntarnos si aprender inglés cambiaría la percepción de la vida de esta población joven, o si más bien fomentaría la confusión y el desasosiego al presentar la sociedad como un gran mercado y el consumismo como la única vía para llegar a participar de esa sociedad-mercado.

En este sentido Pérez, Mora y Morales apuntan que la "exclusión social es mucho más que sinónimo de privaciones, es sinónimo de desempoderamiento" (2006, p. 21). En consecuencia y a razón de ser congruente con los objetivos y compromisos de la educación pública entre ella la universitaria, la enseñanza y aprendizaje del inglés en Costa Rica deben 
facultar y ayudar al individuo a construir su vida con un sentido más allá del que le ofrece la mercadotecnia y el consumismo y así ofrecer herramientas intelectuales que le permitan al individuo leer ese discurso economicista desde una perspectiva más ética.

\section{Pobrezas de afecto y participación}

Las pobrezas de afecto y de participación también plantean la revisión de la o las formas en que docentes - en este caso en enseñanza y aprendizaje del inglés - se aproximan a la cultura joven. Tradicionalmente desde una cultura adulta y antropocéntrica se tiende a reproducir relaciones con altísimos niveles de pobreza de afecto en los que sobresalen los estilos de liderazgo autoritarios dentro del aula y uso de la lengua inglesa. En estos se representa a la gente joven como sujetos carentes, no cognoscentes, inexpertos e inmaduros por lo que se tiende a negarles la participación en diferentes instancias, al mismo tiempo que se alientan prácticas antidemocráticas muy sutiles y otras muy directas.

Primeramente, se observa la selección de estrategias didácticas y técnicas de enseñanza centradas en docentes. Son las personas adultas las que saben usar la lengua y toda manifestación adolescente en la lengua (la jerga, los dichos, la creatividad y la contradicción con las normas), son prohibidas y castigadas, cuando lo que sería más prudente y oportuno en una educación en lengua que tome en cuenta la cultura joven es motivar las sensibilidades lingüísticas hacia el uso y los contextos. Para ello los cursos pueden facilitar espacios para que, al lado del desarrollo de habilidades lingüísticas, se desarrollen esas sensibilidades. Esto es un principio sociolingüístico básico: una persona no habla de igual manera (semántica, sintáctica ni fonológicamente) con todas las personas ni en todos los lugares. Así, en lugar de centrarse en la enseñanza del inglés "estándar" o de los medios y de la academia, vendríamos a proponer la sensibilización lingüística de acuerdo con los contextos o "Networks".

Indiscutiblemente esta propuesta no excluye la variable estándar que se trata de enseñar en la carrera, sino que es un llamado a incluir otros dialectos y variables también muy usadas en la vida cotidiana. En consecuencia, se propone visualizar técnicas de evaluación y de enseñanza que permitan ver la diferencia contextualizada del uso de la lengua. Así por ejemplo, los cursos de laboratorio se enfrentan ante la necesidad de incluir diferentes manifestaciones culturales muy propios de la cultura joven como la música rock, hip-hop, gospel, las series y animados en inglés y, por supuesto, el cine, pero no desde la 
perspectiva del análisis de error ni de la trivia, sino desde la distribución y reconfiguración de significados.

Lo que ha pasado es que la enseñanza del inglés se ha encerrado en un supuesto academicismo donde, por estar en la universidad, se entiende que los procesos educativos están en ese nivel, sin percatarse de que al excluir otras manifestaciones y no tratar tan siquiera de comprenderlas se mutila lo académico de cualquier proceso. Ese adultocentrismo no permite entender que la gran mayoría de estudiantes de las carreras, ya cuando docentes, trabajarán con adolescentes, a quienes históricamente se les ha enseñado un inglés de adultos y no se les ha motivado a sensibilizarse en los aspectos lingüísticos aquí mencionados. Es en este sentido que la idea de Johns (1997, pp. 8-9), es muy oportuna por cuanto

En muchas teorías que proponen al o a la estudiante como centro del proceso, no se reconoce un grupo estándar de formas lingüísticas que sean superiores a otras, ni un corpus lingüístico que sea científicamente predeterminado y apropiado para todas las ocasiones. En su lugar, existen variaciones de lenguaje, de forma, de tópico... que son aceptables... de esta manera, el desarrollo de las habilidades y capacidades académicas (literacy) es principalmente acerca de la construcción individual del significado, no acerca de la forma. (Traducción mía)

Pareciera inevitable, entonces, preguntarse sobre la relación que tienen esas pobrezas de afecto con respecto a los modelos metodológicos de enseñanza y aprendizaje del inglés predilectos en los cursos, la población joven y docentes. Desde esta perspectiva es obligatorio hacer una revisión completa sobre los saberes que se dan como valiosos y enfrentarlos con enfoques pedagógicos y didácticos que sí fomenten prácticas más horizontales e inclusivas. Se debe desarrollar la capacidad de discernir si las metodologías, aun cuando se digan ser comunicativas, promuevan saberes y valores antidemocráticos, intolerantes, clasistas, egocéntricos e irrespetuosos. Dicho de otro modo, la didáctica debe posibilitar un saber más allá que el control de la disciplina estudiantil, el manejo de grupos y la omnisciencia docente.

Consecuentemente, es necesario tomar en cuenta que el trabajo docente y estudiantil no puede reducirse al mero desarrollo de habilidades como si éstas fueran inocentes del impacto que generan en el imaginario colectivo. El desarrollo de habilidades lingüísticas 
debe estar acompañado del desarrollo de sensibilidades socio-culturales porque de otro modo se puede creer que las posiciones ideológicas desarrolladas a través de los discursos - bien comunicados - no son de la competencia del proceso educativo en la enseñanza y el aprendizaje de una lengua, como lo es la inglesa. Cabe re-pensar si es ética y moralmente correcto que un estudiante produzca un discurso verbal racista bien articulado en términos sintácticos y fonológicos, y por esto último debe consignársele una buena nota. Nuevamente, es oportuno preguntarse ¿son el vocabulario, la puntuación, la ortografía, las reglas gramaticales en una composición escrita más importantes que el contenido discursivo de una redacción? (nótese que contenido no sólo se refiere a la noción tradicional de cantidad y coherencias de ideas). Si la respuesta fuera afirmativa entonces los compromisos humanistas de la universidad pública, por ejemplo, serían reduccionismos abordados desde contenidos exóticos exclusivos de las Humanidades o cursos específicos y no una propuesta universitaria articulada.

\section{Consideraciones finales}

La política educativa es política cultural. Cuando las propuestas de desarrollo existentes encierran la educación en el ámbito de la preparación para el trabajo, vemos que la pobreza material representa todo un paradigma; es decir, al ver la pobreza como el problema y al entender la solución de este a partir de la consecución de un trabajo, no queda espacio para objetivizar o subjetivisar otras experiencias, no solamente con respecto a la pobreza sino también con respecto a otros estilos de vida y visones de mundo que resultan alternativas más respetuosas del ser humano y sus entornos, que además no representan al mundo ni a la vida como un gran mercado y al consumismo como la vía para participar en estas.

En el campo de la educación, y por ende en los procesos de enseñanza y aprendizaje del inglés, se utiliza la idea de pobreza como carencia económica para fundamentar las decisiones y convencer sobre lo que es bueno, correcto, necesario y útil en educación. Esta idea, por una parte, alimenta el discurso de que la única forma de enfrentar la pobreza es creando empleos que satisfagan la IED y las necesidades más inmediatas de los sectores industriales y comerciales del país; por otra, permite echar adelante el plan neoliberal de desarticulación de un Estado de bienestar social y solidario, traslapando su razón de ser y responsabilidades al mercado, el cual, mediante el sector privado, asume las tareas que se 
le han conferido al mismo. De esta manera, el sector privado comercializa los servicios públicos que en principio nacen a razón de atender los derechos de los individuos quienes, dentro de la lógica neoliberal, terminan pagando - consumiendo - lo que serían derechos: educación, salud o seguridad. Esto es más que evidente en Costa Rica desde hace un par de décadas, luego de la implementación de los Proyectos de Ajuste Estructural, en que las escuelas, colegios y universidades, las clínicas de salud y las empresas de seguridad todas del sector privado - se han multiplicado exponencialmente, mientras las instituciones estatales encargadas de brindar estos servicios presentan serios problemas estructurales y cada vez tienen más problemas de base política para su fortalecimiento.

Es en este contexto de privatización y mercadotécnica que se simboliza al inglés como un bien de consumo y se le adjudican poderes nunca antes conferidos a esa lengua. Con esto la enseñanza y aprendizaje se reconfiguran en la sociedad costarricense y se les valora en el ámbito laboral. No es errado pensar que saber hablar inglés es un factor deseable y para algunos puestos necesario. Pero este factor, un puesto laboral y un salario, solamente aseguran palear un tipo de pobreza relacionada con la carencia de bienes de consumo. Más aun, a pesar de que se devenguen salarios muy altos se puede estar siempre cerca o por debajo de las líneas de otras pobrezas no económicas, y se puede sufrir de las mismas a nivel de pauperización, como se ha expuesto alrededor de la idea de la pobreza de identidad, situación muy evidente con las pobrezas de protección y de afecto de las que sufrimos diariamente en la vida cotidiana.

Bien es cierto que la universidad pública no es la encargada de atender la pobreza en Costa Rica, pero ésta ha asumido el compromiso de propiciar mejores opciones de vida a la mayor cantidad de individuos posible por medio de la docencia, la investigación y la acción social. Es dentro de esta lógica que se deben entender los quehaceres académicos; dentro de esa búsqueda del bien común, más allá del empleo, donde la consecuencia y compromiso de la excelencia académica se transforman en trabajo como una forma de seguir construyendo la sociedad, no meras formas de satisfacer necesidades básicas o el consumismo, es la vía por la cual se puede trabajar contra diferentes manifestaciones de pobrezas que atentan contra los Derechos Humanos Fundamentales.

Como se ha discutido anteriormente, la idea de pobreza referida a la carencia material y al consumismo es un tecnicismo que imposibilita a la academia trabajar - si se quiere, efectivamente - en aras de lograr el compromiso del bien común, pues en el afán de 
solventar esa pobreza económica se generan otras en áreas muy sensibles aunque tal vez menos visibles. Por esto, asumir una identidad complaciente enfocando el Plan de Bachillerato y licenciatura en la enseñanza del inglés a mercados laborales o necesidades más inmediatas de algún sector empresarial, genera un distanciamiento con respecto a los objetivos y compromiso sociales asumidos por la universidad pública, ya que como lo indican diferentes informes del Estado de la Nación, un mayor crecimiento en la inversión extranjera, la producción y las exportaciones no han redundado en una mejor repartición de los excedentes económicos ni en la mejora de los salarios de los individuos, que para el caso expuesto, sabrían hablar inglés.

No se trata de darle la espalda a los sectores productivos y concentrarse en la búsqueda de alternativas desligadas de estos. La universidad pública y, para el caso, la carrera Bachillerato en la enseñanza del inglés, también debe trabajar en conjunto con los sectores industria y comercio, pero no debe hacerlo asumiendo el pensamiento neoliberal en el que la idea de pobreza, muy cómodamente, se reduce a la carencia de bienes de consumo, si no propiciando un diálogo en el marco de la búsqueda del bien común y el fortalecimiento de los Derechos Humanos Fundamentales, dentro de los cuales se reconfiguren tanto los saberes disciplinarios como los compromisos socioeconómicos de la carrera.

\section{Referencias}

Álvarez, Mariana y Bassi, Horacio. (2010). Habilidades para el siglo XXI: La enseñanza del inglés en Costa Rica. Recuperado en octubre de 2010 de http://www.iadb.org/topics/education/educationandtheidb/index.cfm?language=Sp\&artl $\mathrm{D}=6641$.

Araya Araya, Karla y Córdoba González, Gustavo. (2008). Teacher Knowledge in Language Teaching: An Analysis of its Socio-Historical Construction in the Western Central Region of Costa Rica. Revista Actualidades Investigativas en Educación, 8 (3). Recuperado en marzo de 2009, de http://revista.inie.ucr.ac.cr/buscararticulos/controlador/Article/accion/show/articulo/teacher-knowledge-in-englishlanguage-teaching-an-analysis-of-its-socio-historical-construction-in-the-westerncentral-region-of-costa-rica.html

Araya Araya, Karla y Córdoba González, Gustavo. (2010). Principios epistemológicos y axiológicos del saber docente en la enseñanza del inglés. Revista Actualidades Investigativas en Educación, 10 (3). Recuperado en febrero de 2011, de http://revista.inie.ucr.ac.cr/buscararticulos/controlador/Article/accion/show/articulo/principios-epistemologicos-y- 
$\underline{\text { axiologicos-del-saber-docente-en-la-ensenanza-del-ingles.html }}$

Artigas, Carmen. (2003). La incorporación del concepto de derechos económicos sociales y culturales al trabajo de la CEPAL. Recuperado el 17 de julio de 2009 de http://www.eclac.cl/publicaciones/xml/8/13598/lcl1964 P.pdf

Carr, Philip. (1999). English Phonetics and Phonology. Maryland: Blackwell Publishing.

Castillo Serrano, Deyanira. (1998). Afro-Caribbean Schools in Costa Rica: 1934-1948. Tesis doctoral, University of Texas at Austin, Estados Unidos de Norteamérica.

Castro Valverde, Carlos. (2006). Decimotercer informe Estado de la Nación en Desarrollo Humano Sostenible. San José: Proyecto Estado de La Nación.

Córdoba Cubillo, Patricia, Coto Keith, Rossina y Ramírez Salas, Marlene. (2005). La enseñanza del inglés en Costa Rica y la destreza auditiva en el aula desde una perspectiva histórica. Revista Actualidades Investigativas en Educación 5 (2). Recuperado en marzo de 2009 de http://revista.inie.ucr.ac.cr/buscararticulos/controlador/Article/accion/show/articulo/resena-historica-sobre-la-ensenanzadel-ingles-en-costa-rica-y-el-papel-de-la-destreza-auditiva-en-el-aula.html

Costa Rica, CINDE. (2010). Panorama General sobre la Educación en Costa Rica. Recuperado el 28 de marzo de 2011 de http://www.cinde.org/attachments/149 Educaci\%C3\%B3n\%20en\%20Costa\%20Rica.p df

Costa Rica, Consejo Centroamericano de Procuradores de Derechos Humanos. \& Instituto Interamericano de Derechos Humanos. (2008). Políticas públicas regionales sobre la reducción de la pobreza en Centroamérica y su incidencia en el pleno disfrute de los derechos humanos: informe nacional Costa Rica. Recuperado en abril de 2010 http://www.iidh.ed.cr/BibliotecaWeb/Varios/Documentos/BD 125911109/politicas reduc cion pobreza cr.pdf

Costa Rica, Ministerio de Educación Pública, Ministerio de Comercio Exterior, Ministerio de Economía, Industria y Comercio; Instituto Nacional de Aprendizaje, Coalición Costarricense de Iniciativas de Desarrollo, Proyecto Estrategia Siglo XXI. (Setiembre 2007). Plan Nacional de Inglés. Recuperado 14 octubre 2010 de http://www.competitividad.go.cr/bibliotecaimages/documentos/Plan\%20Nacional\%20de \%20Ingles.pdf)

Chomsky, Avi. (Summer, 1995). Afro-Jamaican Traditions and Labor Organizing on United Fruit Company Plantations in Costa Rica, 1910. Recuperado en abril de 2011 de, http://www.jstor.org/stable/3788591

Crystal, David. (2004). The Language Revolution. Cambridge: Polity Press.

Fairclough, Norman. (2001). Language and Power ( $2^{\text {nd }}$ ed.). London: Longman. 
Hernández Herrero, Annabelle. (2008). El inglés en Costa Rica: Requisito indispensable en un mundo globalizado. Revista Actualidades Investigativas en Educación, 8 (2). Recuperado el 11 de marzo de 2010, de http://revista.inie.ucr.ac.cr/ediciones/controlador/Article/accion/show/articulo/el-inglesen-costa-rica-requisito-indispensable-en-un-mundo-globalizado.html.

Hopenhayn, Martín. (s.f.). La pobreza en conceptos, realidades y políticas: una persperctiva recional con énfasis en minorías étnicas. División de Desarrollo Social CEPAL. Recuperado el 09 de julio de 2011 de http://www.iidh.ed.cr/comunidades/diversidades/docs/div enlinea/Pobreza\%20afros.pd f

Jenkins, Jennifer. (2006). World Englishes: A resource book for students. New York: Routledge.

Jenkins, Jennifer. (2000). The phonology of English as an International Language. Oxford: Oxford University Press

Jiménez, Ronulfo, Céspedes, Víctor Hugo y Arce, Gilberto. (2006). La pobreza en Costa Rica 1987-2004. En Victor Hugo Céspedes y Ronulfo Jiménez (Eds.), III Jornada anual de la Academia de Centroamérica (pp. 59-78). San José: Academia de Centroamérica.

Johns, Ann M. (1997). Text, Role, and Context: Developing Academic Literacy. Cambridge: Cambridge University Press.

Marín Arroyo, Edwin. (2004, Agosto-Diciembre). La enseñanza del inglés en Costa Rica en el siglo XIX: una respuesta al modelo económico. Revista Comunicación, 13, (2), 47-55.

Medrano, Celia, Barillas, Byron y Bonilla, Luis Vidal (Eds.). (2002). Los Derechos Económicos, Sociales y Culturales en la Centroamérica de inicios del siglo XXI: Elementos para el diseño de una estrategia conjunta de organizaciones especialistas en Derechos Humanos y sectores sociales para la defensa de los DESC en Centroamérica. San José, C. R.: CODEHUCA.

Narayan, Deepa, Patel, Raj, Schafft, Kai, Rademacher, Anne y Koch-Schulte, Sarah. (2000). La voz de los pobres: ¿Hay alguien que nos escuche? Recuperado el 17 de setiembre de 2009, de http://siteresources.worldbank.org/INTPOVERTY/Resources/3356421124115102975/1555199-1124115187705/vol1 sp.pdf

Navarro, Dunia y Piñeiro, Milagro. (2009). La Sede de Occidente de la Universidad de Costa Rica ante la demanda de profesionales bilingües en español- inglés. Recuperado el 18 de febrero de 2011, de http://www.latindex.ucr.ac.cr/descargador.php?archivo=intrsds007-09

Pérez Sáinz, Juan Pablo, Mora Salas, Minor y Natalia Morales Aguilar. (2007). Exclusión Social y Pobreza Irreducible: reflexiones desde el caso costarricense. Ponencia preparada para el Decimotercer Informe Estado de la Nación. San José, Programa 
Estado de la Nación. Recuperado el 14 de setiembre de 2008, de http://www.estadonacion.or.cr/images/stories/informes/013/docs/Equidad/Perez-MoraMorales-2007.pdf

Quesada Camacho, Juan Rafael. Educación en Costa Rica 1821 - 1940. San José: EUNED.

Romero, Mayra. (1996, marzo). Desarraigo cultural y pobreza: Aproximación a su estudio. Revista de Ciencias Sociales (71), 7-14.

Salazar Mora, Jorge Mario. (2003). Historia de la educación costarricense. San José: EUNED.

Spence Sharpe, Marva. (2004). El criollo limonense: diglosia o bilingüalismo. Recuperado el 01 de setiembre de 2011, de http://www.intersedes.ucr.ac.cr/images/pdf/articulos/volumen5 numero8 2003/13 Spe $\underline{\text { nce.pdf }}$

Spicker, Paul, Álvarez Leguizamón, Sonia y Gordon, David. (2009). Definiciones de Pobreza: Doce grupos de significados en Pobreza: un glosario internacional. Recuperado de http://bibliotecavirtual.clacso.org.ar/ar/libros/clacso/crop/glosario/06spicker.pdf

Trejos, Juan Diego. (1990). Pobreza y política social en Costa Rica. San José: Editorial Universidad de Costa Rica. 